\title{
Targeting of the Subthalamic Nucleus in Patients with Parkinson's Disease Undergoing Deep Brain Stimulation Surgery
}

\author{
Pepijn van den Munckhof · Maarten Bot · P. Richard Schuurman
}

Received: November 30, 2020 / Accepted: January 20, 2021 / Published online: February 9, 2021

(C) The Author(s) 2021

\begin{abstract}
Precise stereotactic targeting of the dorsolateral motor part of the subthalamic nucleus (STN) is paramount for maximizing clinical effectiveness and preventing side effects of deep brain stimulation (DBS) in patients with advanced Parkinson's disease. With recent developments in magnetic resonance imaging (MRI) techniques, direct targeting of the dorsolateral part of the STN is now feasible, together with visualization of the motor fibers in the nearby internal capsule. However, clinically relevant discrepancies were reported when comparing STN borders on MRI to electrophysiological STN borders during microelectrode recordings (MER). Also, one should take into account the possibility of a 3D inaccuracy of up to $2 \mathrm{~mm}$ of the applied stereotactic technique. Pneumocephalus and image fusion errors may further
\end{abstract}

P. van den Munckhof $(\bowtie) \cdot$ M. Bot .

P. R. Schuurman

Department of Neurosurgery, Amsterdam

University Medical Centers, Academic Medical

Center (AMC), Amsterdam, The Netherlands

e-mail: p.vandenmunckhof@amsterdamumc.nl increase implantation inaccuracy. Even when implantation has been successful, suboptimal lead anchoring on the skull may cause lead migration during follow-up. Meticulous preand intraoperative imaging is therefore indispensable, and so is postoperative imaging when the effects of DBS deteriorate during follow-up. Thus far, most DBS centers employ MRI targeting, multichannel MER, and awake test stimulation in STN surgery, but randomized trials comparing surgery under local versus general anesthesia and additional studies comparing MER-STN borders to high-field MRI-STN may change this clinical practice. Further developments in imaging protocols and improvements in image fusion processes are needed to optimize placement of DBS leads in the dorsolateral motor part of the STN in Parkinson's disease.

Keywords: Deep brain stimulation; Magnetic resonance imaging; Microelectrode recordings; Parkinson's disease; Subthalamic nucleus; Targeting 


\section{Key Summary Points}

Precise targeting of deep brain stimulation (DBS) leads to the dorsolateral motor part of the subthalamic nucleus (STN) is paramount for maximizing clinical effectiveness in Parkinson's disease.

With recent 3- and 7-Tesla MRI, developments such as precise targeting now seems feasible, but several procedural factors negatively influence surgical outcome.

3D inaccuracy of up to $2 \mathrm{~mm}$ of the applied stereotactic technique, pneumocephalus, CT/MRI fusion errors, and suboptimal lead anchoring compromise accurate implantation.

To account for these inaccuracies most DBS centers employ, besides MRI targeting, intraoperative electrophysiological confirmation of STN borders and awake test stimulation.

Future studies comparing electrophysiological STN borders to MRISTN borders and further improvements in image fusion are needed to optimize placement of DBS leads in the motor part of the STN.

\section{DIGITAL FEATURES}

This article is published with digital features, including a summary slide, to facilitate understanding of the article. To view digital features for this article go to https://doi.org/10.6084/ m9.figshare.13615103.

\section{INTRODUCTION}

Deep brain stimulation (DBS) of the subthalamic nucleus (STN) is a highly effective treatment for patients with advanced Parkinson's

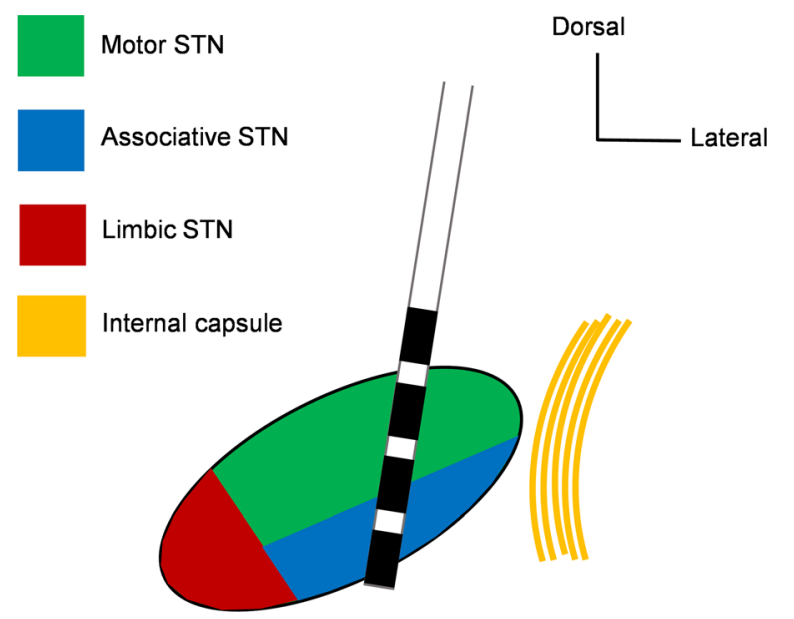

Fig. 1 Coronal representation of the motor, associative, and limbic subdivisions of the human STN. A fourcontact DBS lead is positioned to cover the dorsolateral motor part of the STN, at a safe distance from the limbic STN and internal capsule fibers

disease $[1,2]$. Precise stereotactic targeting of the dorsolateral motor part of the STN is paramount for maximizing clinical effectiveness and preventing side effects [2]. Ideally, the DBS lead should be implanted in the motor area of the STN in such a manner that co-stimulation of the limbic and associative areas of the STN or motor fibers in the nearby internal capsule is minimized (Fig. 1). In daily clinical practice, however, procedural factors influence this ideal targeting strategy. In this review, we describe several of these factors together with recent developments that may improve STN targeting during DBS surgery. This article is based on previously conducted studies and does not contain any new studies with human participants or animals performed by any of the authors.

\section{MAGNETIC RESONANCE IMAGING (MRI) OF THE STN}

Before the MRI era, localization of the STN was performed indirectly, on the basis of an averaged distance from an internal reference that was visualized on a stereotactically acquired ventriculography [3]. The most commonly used reference was the line between the anterior and 

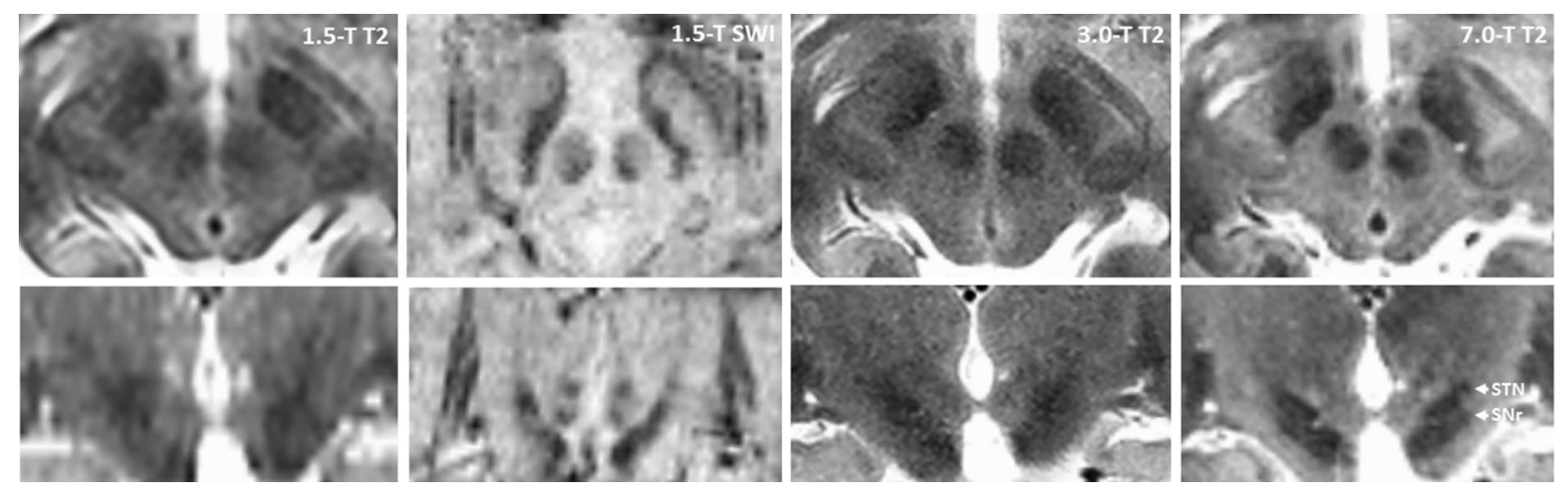

Fig. 2 Axial (upper) and coronal (lower) midbrain sections used for target planning during DBS of the STN on 4 different MRI sequences. On coronal 7-T T2weighted MRI (7.0-T T2, lower right panel), the STN can

be distinguished from surrounding white matter and the more ventrally located substantia nigra pars reticulata (SNr). SWI susceptibility-weighted imaging

posterior commissures (the AC-PC line). Standard human brain atlases were used to determine the position of the subthalamic nucleus, relative to the AC-PC midpoint, in each direction of a Cartesian coordinate system superimposed on the brain by a stereotactic frame. In 1995, Benabid was the first to target the STN using direct visualization on a T2-weighted 1.5$T$ (Tesla) MRI [4]. In recent years, new MRI sequences such as susceptibility-weighted imaging (SWI) have been introduced and magnetic field strengths have increased up to $7 \mathrm{~T}$, all leading to improved STN visualization $[5,6]$. On coronal 7-T T2-weighted imaging, the STN can be distuingished from surrounding white matter and the more ventrally located substantia nigra pars reticulata (SNr) (Fig. 2). Direct targeting of the dorsolateral part of the STN on

MRI is now feasible, together with visualization of the motor fibers in the nearby internal capsule through the use of diffusion tensor imaging (DTI) (Fig. 3).

Notwithstanding these promising developments, one should keep in mind that STN visualization on MRI in fact represents delineation of an iron-dense structure that is thought to overlap with the electrophysiological STN. But how perfect is this overlap? To address this issue, we and others compared STN representation on MRI with the location of the electrophysiological STN as determined by microelectrode recordings (MER) during DBS surgery (see also Sect. "Microelectrode Recordings"). At the calculated stereotactic target depth, the percentage of microelectrodes measuring typical STN activity that were located
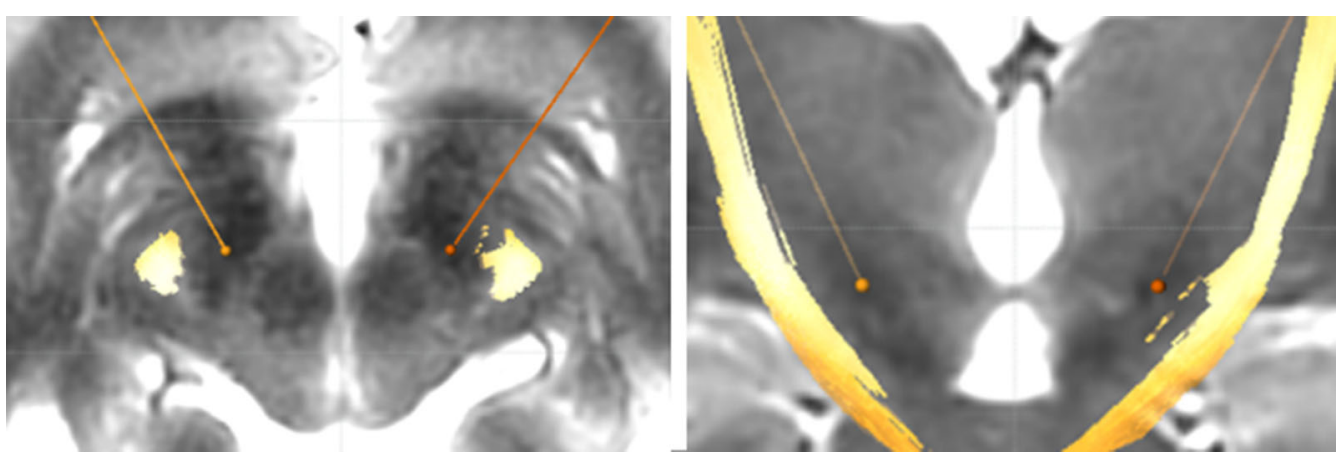

Fig. 3 Axial (left) and coronal (right) midbrain section used for target planning during DBS of the STN on 3.0-T T2weighted MRI, with superimposed diffusion tensor imaging (yellow) of the motor fibers in the internal capsule 
inside STN contour representation on MRI was very high for 1.5-T T2 (99\%) and 3-T T2 (100\%). But for 1.5-T SWI, one out of five microelectrodes measuring STN activity were located outside, mostly lateral of the STN contour [5]. SWI thus does not correctly display the lateral part of the STN in all patients, and SWI-based STN targeting may carry the risk of targeting the STN too medially (i.e., limbic/associative STN). Discrepancies are also found when comparing dorsal/ventral STN borders on MRI to electrophysiological STN. Employing 3-T T2 and SWI, Bus et al. showed that MER-STN started and ended more dorsally than respective dorsal and ventral MRI-STN borders [7]. The largest difference for the dorsal border was found using SWI (on average $2.5 \mathrm{~mm}$ ). Bot et al. used 7-T T2 to show that MER-STN started more ventrally than the dorsal MRI-STN border, on average $0.9 \mathrm{~mm}$ [6]. Whether 3-T T2/SWI and 7-T T2 truly generate opposite results when comparing dorsal MER-STN to dorsal MRI-STN borders remains to be further studied, but it is clear from these findings that STN representation on MRI provides an indication but not an exact representation of the electrophysiological STN boundaries. Thus, relying solely on MRI for DBS lead placement could potentially lead to missing the electrophysiological dorsolateral STN.

\section{ACCURACY OF THE STEREOTACTIC TECHNIQUE}

For more than 70 years, stereotactic procedures are performed with head-mounted stereotactic frames. It provides a stable platform and offers a high degree of accuracy. However, application inaccuracies do occur. The total error, usually expressed as the 3D (euclidean) distance between the stereotactic ( $x, y$, and $z$ ) coordinates of the tip of the DBS lead and those of the intended target, comprises errors associated with each procedural step, including imaging, target selection, vector calculations, and the mechanical errors of the applied stereotactic technique. In 1994, Maciunas et al. reported on more than 21,500 independent accuracy test measurements of a test phantom made with 11,000 computed tomography (CT) images and employing four commonly used stereotactic devices: the Brown-Roberts-Wells (BRW) frame, the Cosman-Roberts-Wells (CRW) frame, the Kelly-Goers Compass frame, and the Leksell frame (Table 1) [8]. In recent years, several groups have reported on inaccuracies during DBS procedures, including groups employing frameless systems and robot-assisted procedures (Table 1) [9-21]. On the basis of these results, one should take into account the possibility of a $3 \mathrm{D}$ inaccuracy of up to $2 \mathrm{~mm}$ of the applied stereotactic technique when targeting the dorsolateral STN, underlining the need for intraoperative imaging to verify lead localization.

\section{INTRAOPERATIVE BRAIN SHIFT}

An underlying assumption of stereotactic neurosurgery is that anatomic structures do not move between preoperative image acquisition and surgery, during surgery, and postoperatively. Cerebrospinal fluid (CSF) loss and subdural air invasion, however, may cause shift of cortical and deeply located brain structures. Several studies reported on pneumocephalusinduced posterior displacement of the $\mathrm{AC}$ and nearby structures such as the STN, in some cases up to $4 \mathrm{~mm}[22,23]$. Moreover, the effects of intracranial air may compromise stereotactic accuracy especially on the second side [24]. In addition to the effects of pneumocephalus on intraoperative localization of the STN, it may also affect follow-up: while the pneumocephalus resolves the brain returns to its original position, thereby potentially displacing DBS leads (Fig. 4). We showed in previous work that DBS leads are prone to anterior bending and concomitant upward displacement along the trajectory of (on average) $3 \mathrm{~mm}$ during longterm follow-up, with the amount of displacement correlating with the amount of pneumocephalus [25]. Such lead displacement may influence the choice of active contact on the DBS lead and settings of the DBS parameters during the first weeks/months of follow-up. To prevent pneumocephalus, CSF loss and subdural air invasion should be minimized by planning burr holes on top of a gyrus so that brain tissue is expected to pack the burr hole from 


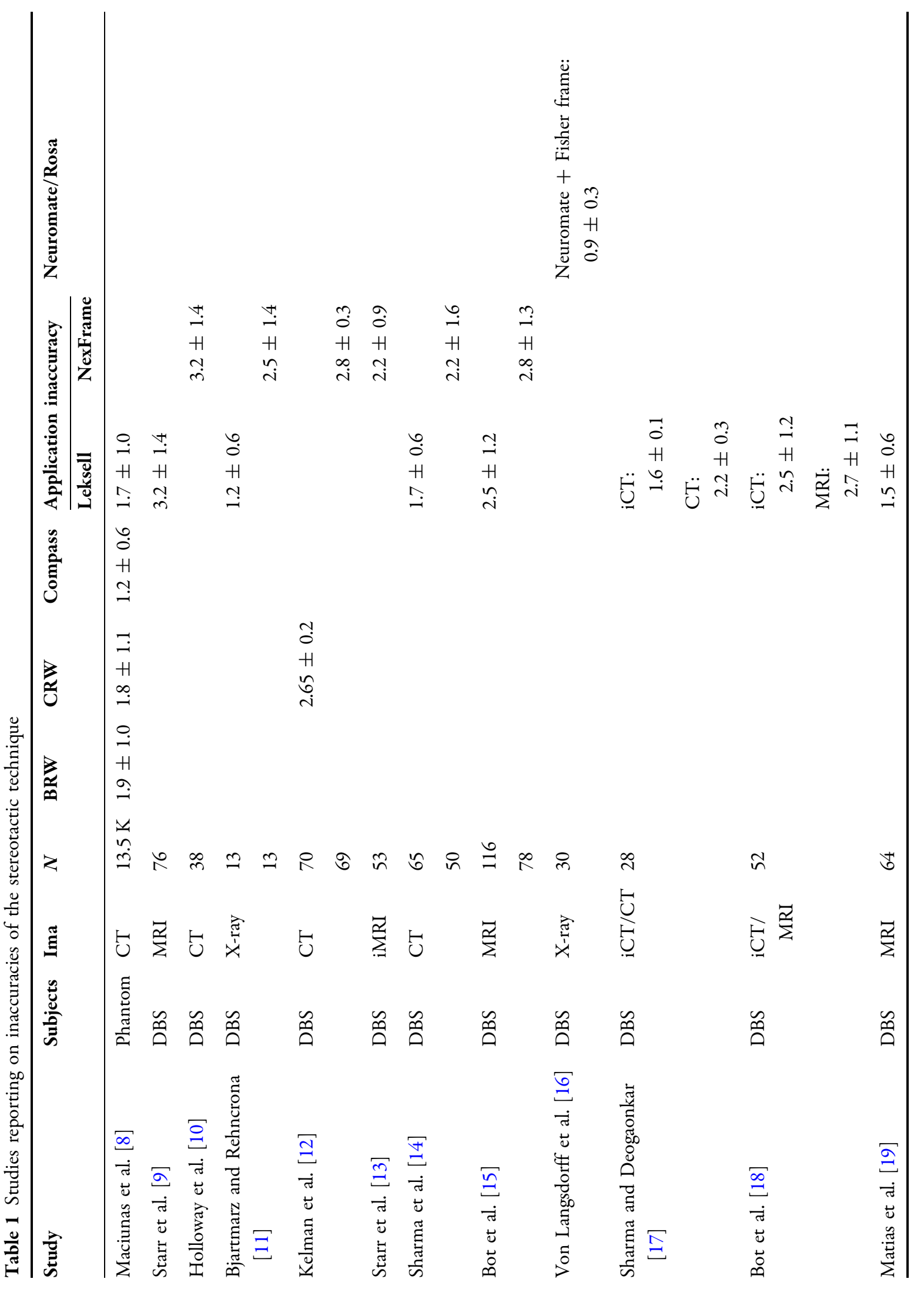




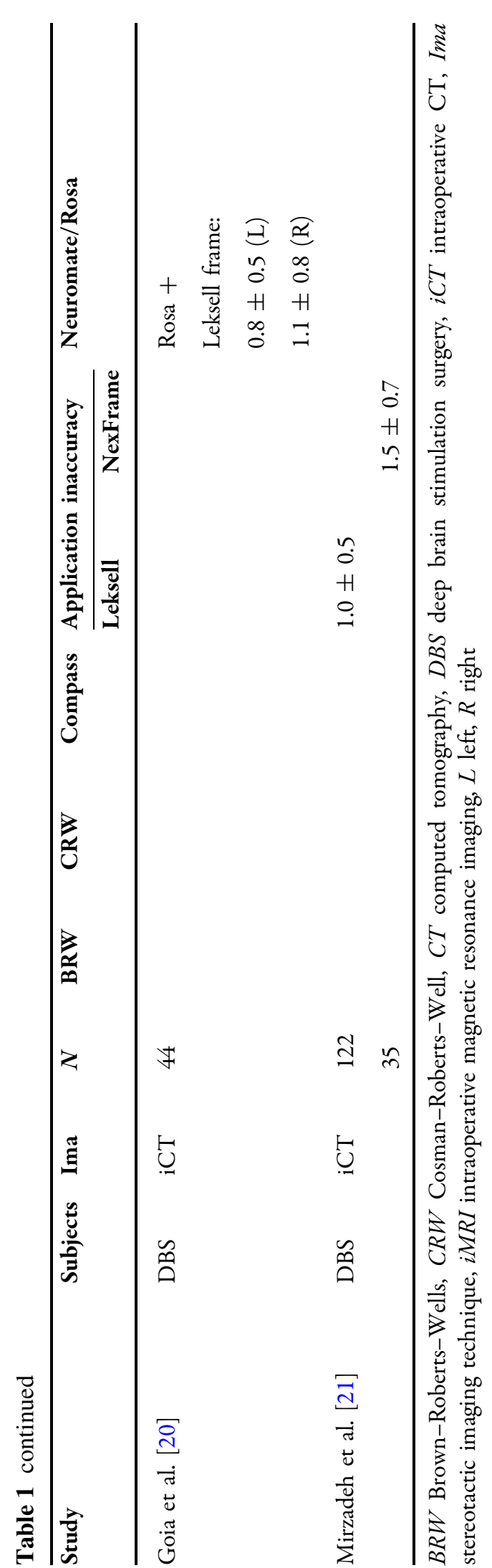

inside during DBS surgery, and by closing burr holes with fibrin glue or bone wax after introduction of the microelectrode(s)/macroelectrode. The preferred position of the patient during DBS surgery in the context of minimizing CSF loss is a matter of ongoing debate. There may be less spontaneous CSF outflow in the semi-sitting compared to supine position. In the supine position, on the other hand, the brain tissue may pack the burr hole from inside more effectively compared to the semi-sitting position, during which the brain "sinks" downward. In addition, the supine position may match the location of brain structures on pre-operative MRI more accurately. However, the supine position may not be as well tolerated as the semi-sitting position during awake surgery. Future studies comparing the supine and semisitting position during deep brain stimulation surgery with respect to these factors are needed.

\section{INTRA/POSTOPERATIVE IMAGING AND IMAGE FUSION}

As stated previously, intra- and postoperative imaging is used to verify DBS lead placement. However, the accuracy of both MRI and CT in lead localization is still controversial. The diameter of DBS leads is $1.27 \mathrm{~mm}$, but the size of the lead artifact typically measures $3.6 \mathrm{~mm}$ on MRI and $3.3 \mathrm{~mm}$ on CT (Fig. 5) [26, 27]. The issue whether the exact position of the actual DBS lead within the MRI artifact is concentric or eccentric was studied by Hyam et al. in patients in whom DBS leads were explanted as a result of infection or suboptimal efficacy. By comparing the center of the lead hypointensity on postimplantation MRI to the center of the lead track on postexplantation MRI, the authors were able to calculate a mean difference in $x$ coordinate of $0.4 \mathrm{~mm}$, and in $y$ coordinate of $0.6 \mathrm{~mm}$ (the $x-y$ vectorial difference averaged $0.7 \mathrm{~mm}$ ) [28]. Because of this small difference, it seems reasonable to assume that the lead hypointensity seen on postimplantation MRI is close to concentric. A similar study on the exact position of the DBS lead within the CT artifact is probably not feasible, since the contrast 


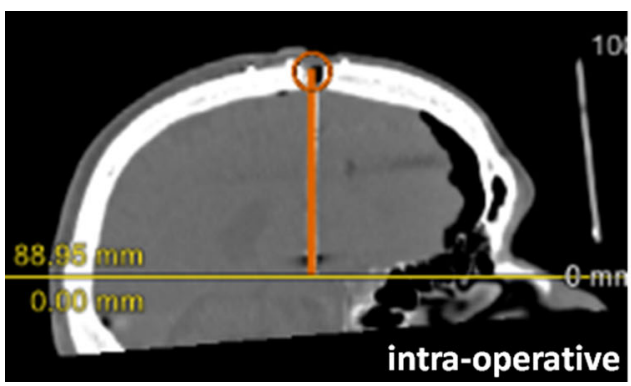

Fig. 4 Intraoperative (left panel) and 1-year follow-up (right panel) analysis of DBS lead on semi-sagittal CT reconstructions parallel to the lead. Intraoperative CT shows pneumocephalus, posteriorly shifted frontal cortex,

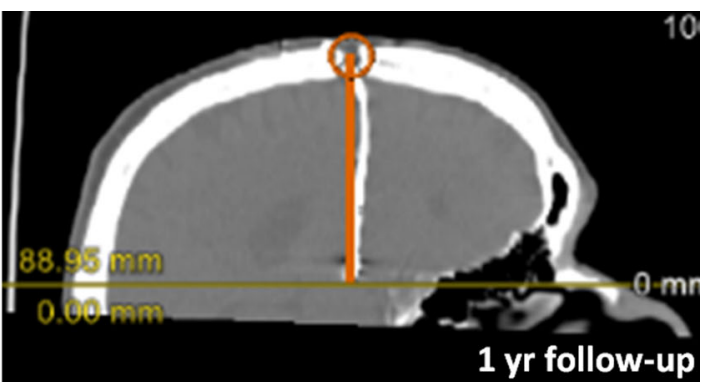

and a straight lead trajectory. Follow-up CT shows resolved pneumocephalus and anterior bending of the DBS lead
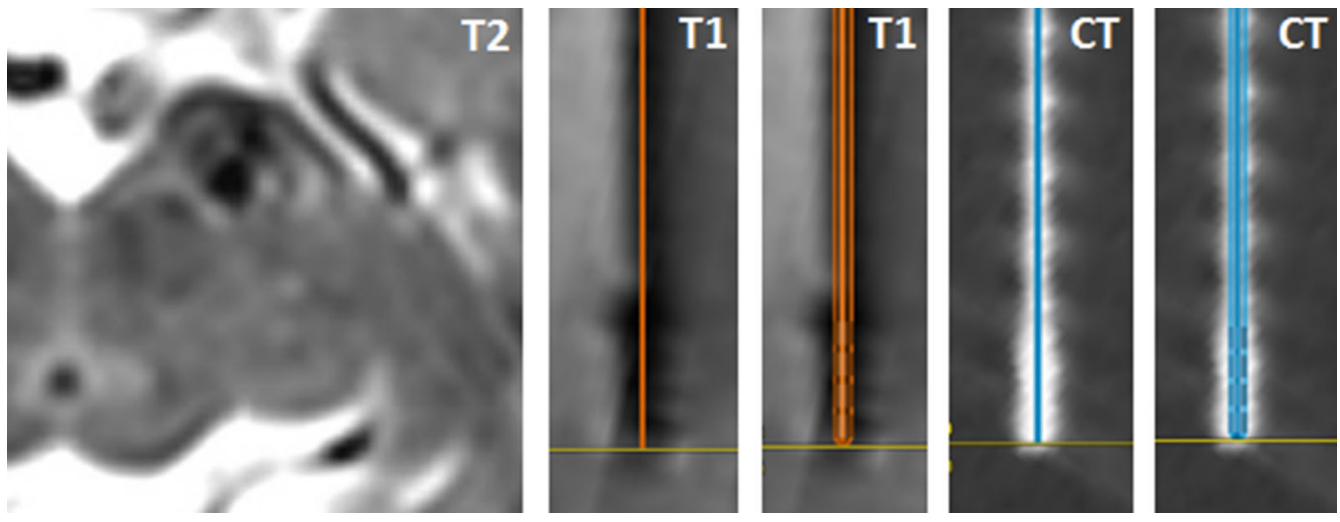

Fig. 5 Axial 1.5-T T2 of left midbrain showing typical $\pm 3.5 \mathrm{~mm}$ diameter artifact of DBS lead (left panel). Semi-sagittal T1 and CT reconstructions parallel to the lead (four panels on the right) show that the diameter of

resolution of CT seems insufficient to correctly display the lead track on postexplantation CT.

Another factor at play is the software used for co-registration of the pre-, intra-, and postoperative imaging. Engelhardt et al. reported a small but significant 3D difference (on average $1.2 \mathrm{~mm}$ ) in the coordinates of the deepest lead contact when calculated on four different commercially available co-registration devices [29]. Furthermore, additional image co-registration errors may occur between two different scan modalities: O'Gorman et al. compared lead coordinates on postoperative stereotactic CT with lead coordinates on postoperative CT coregistered with preoperative stereotactic MRI the lead artifact on MRI $(3.6 \mathrm{~mm})$ and CT $(3.3 \mathrm{~mm})$ is considerably thicker than the actual diameter of the DBS lead $(1.3 \mathrm{~mm})$

using four different co-registration software programs and reported a mean 3D discrepancy of 1.2 to $1.7 \mathrm{~mm}$ (the $x-y$ vectorial discrepancy averaged 0.6 up to $0.7 \mathrm{~mm}$ ) [30]. The use of coregistration software and the choice of different scan modalities thus influences the observed location of the DBS lead.

\section{MICROELECTRODE RECORDINGS}

STN neurons in patients with Parkinson's disease have characteristic firing rates in irregular bursts, which have movement- and tremor-related activity [31]. Burst frequencies of $5-20 \mathrm{~Hz}$ are observed in the dorsal STN, while activity 
deeper into the STN shifts to $15-40 \mathrm{~Hz}$ frequencies [32]. Many DBS centers therefore perform intraoperative electrophysiological measurements to localize the dorsolateral motor part of the STN. Currently, MER remains the electrophysiological technique most commonly used. During a typical exploratory MER track starting just above the STN, a low background noise and absence of single cell recordings is followed by a sudden increase in background activity and irregular bursts of spontaneously active neurons when the MER tip enters the dorsolateral STN. Within the dorsolateral STN, MER may be used to check for the presence of kinesthetic (or movement-related) cells, i.e., neurons that respond to passive movement by changing their firing rate and pattern [33]. At the ventral STN border, the exit of the MER tip out of the STN corresponds to a sudden decrease in background noise and loss of single cell activity. Neuronal activity may then increase again when the electrode tip enters the $\mathrm{SNr}$, although its neurons are more sparse and fire more regularly than STN neurons [34]. To increase the chances of targeting the dorsolateral STN, given the occurrence of imaging and surgical inaccuracies, Benabid developed a MER holder with five MER channels, with the central channel aiming at the planned MRI target, with additional anterior, lateral, posterior, and medial channels at a 2-mm distance from the central channel [34]. In 2010, we reported how often each of these five MER channels was chosen for final STN DBS lead implantation: the central channel was chosen in $50 \%$ of the cases, the anterior channel in $24 \%$, the lateral channel in $10 \%$, the posterior channel in $10 \%$, and the medial channel in $6 \%$ [35]. Because of these results and the finding of a systematic literature review showing that the number of MER penetrations may increase the risk of intracerebral hemorrhage [36], we reduced the number of MER channels in the years thereafter to three (MRI target, $2 \mathrm{~mm}$ anterior and $2 \mathrm{~mm}$ lateral channels) and found that $92 \%$ of final STN DBS leads were indeed implanted in one of those three channels [37]. Three-channel instead of five-channel MER thus provides sufficient accuracy to target the dorsolateral STN in the large majority of patients.
The use of MER may be associated with an increased risk of hemorrhage and several groups reported good clinical outcome in MRI-guided STN DBS without the use of MER [38, 39]. Do we really need MER during STN DBS surgery? Can one rely solely on MRI guidance? As stated previously, not all MRI sequences properly display the dorsolateral STN, and clinically relevant discrepancies were reported when comparing dorsal STN borders on MRI to the electrophysiological dorsal STN border. Indeed, in our 2020 study no electrophysiological STN signal was found in 5\% of the MRI-targeted trajectories, and unexpectedly short STN MER trajectories ( $2.5 \mathrm{~mm}$ or less) were seen in $13 \%$ of MRI-targeted channels [37]. Similarly, Lozano et al. reported a $20 \%$ mismatch between the expected STN on 1.5-T MRI and the MER findings, with mismatch defined as an STN MER trajectory of less than $4 \mathrm{~mm}$ [40]. Notably, in our 2020 study the $x-y$ vectorial stereotactic inaccuracy among MRI-targeted trajectories with $2.5 \mathrm{~mm}$ or less STN MER activity exceeded the mean stereotactic error (of $1.25 \mathrm{~mm}$ ) more often than the inaccuracy among MRI-targeted trajectories with more than $2.5 \mathrm{~mm}$ STN MER activity (67\% versus $42 \%$, respectively). Besides the ability of MER to properly identify the dorsolateral STN, it also supplies important intraoperative feedback on the accuracy (or lack thereof) of the stereotactic method, when changes can still be made. If one decides to omit MER and rely on MRI guidance only, accurate and reliable intraoperative imaging to check stereotactic accuracy thus seems indispensable. Future studies comparing STN borders on ultrahigh field MRI to the electrophysiological STN during DBS surgery with verified ultra-low intraoperative stereotactic inaccuracy are needed to determine whether MRI can really replace MER in localizing the dorsolateral STN. Alternatively, intraoperative spectral analysis of local field potentials (LFPs) while descending the final DBS lead into the STN may also provide neurophysiological information relevant to the position of the lead relative to the dorsolateral STN [41]. Also, MRI techniques such as tractography and connectivity mapping provide additional insight into STN subdivisions, and may replace the need for electrophysiological 
motor STN identification [42, 43]. However, one should realize that (hard to avoid) stereotactic and image-fusion-related inaccuracies carry the risk of "missing" the dorsolateral motor part of the STN when one decides to use MRI guidance only.

\section{AWAKE TEST STIMULATION}

Many DBS centers conduct test stimulation in awake patients to localize that part of the STN where maximal motor improvement at low stimulation intensities and high thresholds for stimulation-induced side effects are observed. Furthermore, intraoperative testing can reduce the time needed for postoperative programming by identifying the contacts most likely to be selected [44]. Recent developments in MRI visualization of the dorsolateral STN and motor fibers in the nearby internal capsule, however, could make awake test stimulation redundant, allowing for DBS surgery under general anesthesia. Asleep DBS would offer a major advantage for patient comfort and surgical workflow. Thus far, several groups reported good clinical outcomes in patients with Parkinson's disease undergoing STN DBS surgery under general anesthesia [45-47]. In our previously mentioned 2020 study employing MRI targeting, three-channel MER, and awake test stimulation, we retrospectively determined the relative contribution of awake test stimulation in STN DBS lead placement by analyzing where final DBS leads were implanted and why these locations were chosen [37]. For final lead placement, the MRI-targeted trajectory was chosen in 39\% of STNs (18\% of bilaterally implanted patients), the trajectory with the longest STN MER signal in $60 \%$ of STNs $(38 \%$ of bilaterally implanted patients). The most frequently noted reason why another trajectory was chosen for final lead placement was a lower threshold for side effects in the planned trajectory. Interestingly, a superior effect of test stimulation on PD symptoms was never noted as the reason, suggesting that test stimulation in MRI-targeted, anterior and lateral channels was equally effective. Alternatively, spontaneous improvement of parkinsonian motor symptoms following penetration of the STN, referred to as the microlesion effect, may have flattened the difference in stimulation-induced motor improvement among the different channels [48]. Most observed side effects that influenced the choice of trajectory for final lead placement in our 2020 study were thought to originate from co-stimulation of the nearby internal capsule [37]. However, the clinical relevance of the observed side effects and of the stimulation threshold at which they occurred were not prospectively studied, so it is not possible to deduce from these retrospective data whether the decision not to implant the final lead in a certain trajectory led to better outcomes. Evidently a trajectory in which mouth contraction or dysarthria occurred at $2 \mathrm{~mA}$ seems even in retrospect not a good choice for final DBS lead placement. But a trajectory in which gaze paresis occurs at $5 \mathrm{~mA}$ might still have been a good choice for final lead placement, since it is not common to employ STN DBS above $4.5 \mathrm{~mA}$.

To investigate whether awake test stimulation during STN DBS surgery is really indispensable, we started a controlled trial in 2017 randomizing 110 patients between surgery under local or general anesthesia (GALAXY trial) [49]. Of note, we will perform MER in all patients in order to optimize lead implantation in the electrophysiological dorsolateral STN in both the awake and asleep study group. Reduction of propofol levels during MER allows for the detection of typical STN neuronal firing patterns [50]. The results of the GALAXY trial are expected to be reported by the end of 2020 .

\section{DBS LEAD ANCHORING TECHNIQUES}

Various methods have been developed to secure the DBS lead to the skull, such as titanium microplates, acrylic cement, and several commercially available plastic cap systems. For all techniques, lead migration has been reported. In a comparative study, we observed less longterm (1 year) lead migration for leads anchored with the commercial plastic cap system $(1.5 \mathrm{~mm})$ than for leads anchored with a titanium microplate $(2.3 \mathrm{~mm})$ [51]. But even with 
such commercial plastic cap systems, Morishita et al. reported lead migration of more than $3 \mathrm{~mm}$ in $12 \%$ of their DBS patients at 6 -month follow-up [52]. Similar to pneumocephalus, anchoring-related lead displacement may influence the choice of active contact on the DBS lead and settings of the DBS parameters during follow-up. Repeated postoperative imaging to accurately determine the location of lead contacts is therefore strongly advocated when the effects of DBS deteriorate during follow-up. Finally, we usually place at least one stimulation contact of the multiple-contact electrodes below the optimal stimulation point as determined during surgery, so that it is possible to switch to a lower contact point in case of upward displacement after longer-term follow-up.

\section{CONCLUSIONS}

The underlying idea of STN DBS in patients with advanced PD is quite simple: DBS leads are to be implanted in the dorsolateral motor part of the STN, at a safe distance from its other subdivisions and nearby internal capsule fibers. However, several factors complicate this ideal targeting strategy. Not all MRI sequences properly display the dorsolateral STN, and clinically relevant discrepancies were reported when comparing STN borders on MRI to electrophysiological STN borders. The 3D inaccuracy of stereotactic techniques averages $2 \mathrm{~mm}$. Pneumocephalus and image fusion techniques may further increase implantation errors. Even when implantation has been successful, suboptimal lead anchoring on the skull may cause lead migration during follow-up. Meticulous preand intraoperative imaging is evidently indispensable, and so is postoperative imaging when the effects of DBS deteriorate during follow-up. Thus far, most DBS centers employ MRI targeting, multichannel MER, and awake test stimulation in STN surgery, but ongoing and future studies may change this clinical practice. The soon to be reported results of the GALAXY trial randomizing patients between MER-guided surgery under local or general anesthesia will tell whether awake test stimulation during STN
DBS surgery is indispensable, Furthermore, studies comparing MER (or LFP)-STN borders during DBS surgery with verified intraoperative ultra-low stereotactic inaccuracy to MRI-STN borders may serve to study whether MER is redundant during 7-T MRI-guided STN surgery. Finally, developments in imaging protocols and improvements in image fusion processes are needed to further optimize placement of DBS leads in the dorsolateral motor part of the STN in Parkinson's disease.

\section{ACKNOWLEDGEMENTS}

Funding. No funding or sponsorship was received for this study or publication of this article.

Authorship. All named authors meet the International Committee of Medical Journal Editors (ICMJE) criteria for authorship for this article, take responsibility for the integrity of the work as a whole, and have given their approval for this version to be published.

Disclosures. The DBS team of the AMC received unrestricted research grants from Medtronic and received financial compensation for teaching courses for the European Continue Medical Training program. Pepijn van den Munckhof and Maarten Bot received travel grants from Medtronic. P. Richard Schuurman acts as an independent advisor for Boston Scientific, Elekta and Medtronic.

Compliance with Ethics Guidelines. This article is based on previously conducted studies and does not contain any new studies with human participants or animals performed by any of the authors.

Open Access. This article is licensed under a Creative Commons Attribution-NonCommercial 4.0 International License, which permits any non-commercial use, sharing, adaptation, distribution and reproduction in any medium or format, as long as you give appropriate credit to the original author(s) and the source, provide 
a link to the Creative Commons licence, and indicate if changes were made. The images or other third party material in this article are included in the article's Creative Commons licence, unless indicated otherwise in a credit line to the material. If material is not included in the article's Creative Commons licence and your intended use is not permitted by statutory regulation or exceeds the permitted use, you will need to obtain permission directly from the copyright holder. To view a copy of this licence, visit http://creativecommons.org/licenses/by$\mathrm{nc} / 4.0 /$.

\section{REFERENCES}

1. Odekerken VJ, van Laar T, Staal MJ, et al. Subthalamic nucleus versus globus pallidus bilateral deep brain stimulation for advanced Parkinson's disease (NSTAPS study): a randomised controlled trail. Lancet Neurol. 2013;12:37-44.

2. Benabid AL, Chabardes S, Mitrofanis J, Pollak P. Deep brain stimulation of the subthalamic nucleus for the treatment of Parkinson's disease. Lancet Neurol. 2009;8:67-81.

3. Schaltenbrand G, Bailey P, editors. Introduction to stereotaxis with an atlas of the human brain. Stuttgart: George Thieme Verlag; 1959.

4. Limousin P, Pollak P, Benazzouz A, et al. Effect on parkinsonian signs and symptoms of bilateral subthalamic nucleus stimulation. Lancet. 1995;345: 91-5.

5. Bot M, Bour L, de Bie RM, Contarino MF, Schuurman PR, van den Munckhof P. Can we rely on susceptibility-weighted imaging for subthalamic nucleus identification in deep brain stimulation surgery? Neurosurgery. 2016;78:353-60.

6. Bot $\mathrm{M}$, Verhagen $\mathrm{O}$, Caan $\mathrm{M}$, et al. Defining the dorsal STN border using 7.0-T MRI: a comparison to microelectrode recordings and lower field strength MRI. Stereotact Funct Neurosurg. 2019;97:153-9.

7. Bus S, van den Munckhof P, Bot M, et al. Borders of STN determined by MRI versus the electrophysiological STN. A comparison using intraoperative CT. Acta Neuochir (Wien). 2018;160:373-83.

8. Maciunas RJ, Galloway RL Jr, Latimer JW. The application accuracy of stereotactic frames. Neurosurgery. 1994;35:682-94.
9. Starr PA, Christine CW, Theodosopoulos PV, et al. Implantation of deep brain stimulators into the subthalamic nucleus: technical approach and magnetic resonance imaging-verified lead locations. J Neurosurg. 2002;97:370-87.

10. Holloway KL, Gaede SE, Starr PA, Rosenow JM, Ramakrishnan V, Henderson JM. Frameless stereotaxy using bone fiducial markers for deep brain stimulation. J Neurosurg. 2005;103:404-13.

11. Bjartmarz H, Rehncrona S. Comparison of accuracy and precision between frame-based and frameless stereotactic navigation for deep brain stimulation electrode implantation. Stereotact Funct Neurosurg. 2007;85:235-42.

12. Kelman C, Ramakrishnan V, Davies A, Holloway K. Analysis of stereotactic accuracy of the CosmanRobert-Wells frame and Nexframe frameless systems in deep brain stimulation surgery. Stereotact Funct Neurosurg. 2010;88:288-95.

13. Starr PA, Martin AJ, Ostrem JL, Talke P, Levesque N, Larson PS. Subthalamic nucleus deep brain stimulator placement using high-field interventional magnetic resonance imaging and a skull-mounted aiming device: technique and application accuracy. J Neurosurg. 2010;112:479-90.

14. Sharma M, Rhiew R, Deogaonkar M, Rezai A, Boulis $\mathrm{N}$. Accuracy and precision of targeting using frameless stereotactic system in deep brain stimulator implantation surgery. Neurol India. 2014;62: 503-9.

15. Bot $M$, van den Munckhof $P$, Bakay R, Sierens D, Stebbins G, Verhagen ML. Analysis of stereotactic accuracy in patients undergoing deep brain stimulation using Nexframe and the Leksell frame. Stereotact Funct Neurosurg. 2015;93:316-25.

16. Von Langsdorff D, Paquis P, Fontaine D. In vivo measurement of the frame-based application accuracy of the Neuromate neurosurgical robot. J Neurosurg. 2015;122:191-4.

17. Sharma M, Deogaonkar M. Accuracy and afety of targeting using intraoperative "O-arm" during placement of deep brain stimulation electrodes without electrophysiological recordings. J Clin Neurosci. 2016;27:80-6.

18. Bot M, van den Munckhof P, Bakay R, Stebbins G, Verhagen ML. Accuracy of intraoperative computed tomography during deep brain stimulatio procedures: comparison with postoperative magnetic resonance imaging. Stereotact Funct Neurosurg. 2017;95:183-8.

19. Matias CM, Frizon LA, Nagel SJ, Lobel DA, Machado AG. Deep brain stimulation outcomes in patients 
implanted under general anesthesia with framebased stereotaxy and intraoperative MRI. J Neurosurg. 2018;129:1572-8.

20. Goia A, Gilard V, Lefaucheur R, Welter ML, Maltête D, Derrey S. Accuracy of the robot-assisted procedure in deep brain stimulation. Int J Med Robot. 2019;15:e2032.

21. Mirzadeh Z, Chen T, Chapple KM, et al. Procedural variables influencing stereotactic accuracy and efficiency in deep brain stimulation surgery. Oper Neurosurg (Hagerstown). 2019;17:70-8.

22. Miyagi Y, Shima F, Sasaki T. Brain shift: an error factor during implantation of deep brain stimulation electrodes. J Neurosurg. 2007;107:989-97.

23. Khan MF, Mewes K, Gross RE, Skrinjar O. Assessment of brain shift related to deep brain stimulation surgery. Stereotact Funct Neurosurg. 2008;86: 44-53.

24. Azmi H, Machado A, Deogaonkar M, Rezai A. Intracranial air correlates with preoperative cerebral atrophy and stereotactic error during bilateral STN DBS surgery for Parkinson's disease. Stereotact Funct Neurosurg. 2011;89:246-52.

25. Van den Munckhof $\mathrm{P}$, Contarino MF, Bour LJ, Speelman JD, de Bie RM, Schuurman PR. Postoperative curving and upward displacement of deep brain stimulation electrodes caused by brain shift. Neurosurgery. 2010;67:49-53.

26. Pollo C, Villemure JG, Vingerhoets F, Ghika J, Maeder P, Meuli R. Magnetic resonance artifact induced by the electrode Activa 3389: an in vitro and in vivo study. Acta Neurochir (Wien). 2004;146:161-4.

27. Hemm S, Coste J, Gabrillargues J, et al. Contact position analysis of deep brain stimulation electrodes on post-operative CT images. Acta Neurochir (Wien). 2009;151:823-9.

28. Hyam JA, Akram H, Foltynie T, Limousin P, Hariz M, Zrinzo L. What you see is what you get: lead location within deep brain structures is accurately depicted by stereotactic magnetic resonance imaging. Neurosurgery. 2015;11(Suppl 3):412-9.

29. Engelhardt J, Guehl D, Damon-Perrière N, Branchard O, Burbaud P, Cuny E. Localization of deep brain stimulation electrode by image registration is software dependent: a comparative study between four widely used software programs. Stereotact Funct Neurosurg. 2018;96:364-9.

30. O'Gorman RL, Jarosz JM, Samuel M, Clugh C, Selway RP, Ashkan K. CT/MR image fusion in the postoperative assessment of electrodes implanted for deep brain stimulation. Stereotact Funct Neurosurg. 2009;87:205-10.

31. Hutchison WD, Allan RJ, Opitz H, et al. Neurophysiological identification of the subthalamic nucleus in surgery for Parkinson's disease. Ann Neurol. 1998;44:622-8.

32. Dolan K, Martens HC, Schuurman PR, Bour LJ. Automatic noise-level detection for extra-cellular micro-electrode recordings. Med Biol Eng Comput. 2009;47:791-800.

33. Tankus A, Strauss I, Gurevich T, et al. Subthalamic neurons encode both single- and multi-limb movements in Parkinson's disease patients. Sci Rep. 2017;7:42467.

34. Benazzouz A, Breit S, Koudsie A, Pollak P, Krack P, Benabid AL. Intraoperative microrecordings of the subthalamic nucleus in Parkinson's disease. Mov Disord. 2002;17(Suppl 3):S145-9.

35. Bour LJ, Contarino MF, Foncke EM, et al. Long-term experience with intraoperative microrecording during DBS neurosurgery in STN and GPi. Acta Neurochir (Wien). 2010;152:2069-77.

36. Zrinzo L, Foltynie T, Limousin P, Hariz MI. Reducing hemorrhagic complications in functional neurosurgery: a large case series and systematic literature review. J Neurosurg. 2012;116:84-94.

37. Frequin HL, Bot M, Dilai J, et al. Relative contribution of magnetic resonance imaging, microelectrode recordings, and awake test stimulation in finale lead placement during deep brain stimulation surgery of the subthalamic nucleus in Parkinson's disease. Stereotact Funct Neurosurg. 2020. https://doi.org/10.1159/000505710.

38. Aviles-Olmos I, Kefalopoulou Z, Tripoliti E, et al. Long-term outcome of subthalamic nucleus deep brain stimulation for Parkinson's disease using an MRI-guided and MRI-verified approach. J Neurol Neurosurg Psychiatry. 2014;85:1419-25.

39. Lee PS, Weiner GM, Corson D, et al. Outcomes of interventional-MRI versus microelectrode recording-guided subthalamic nucleus deep brain stimulation. Front Neurol. 2018;9:241.

40. Lozano CS, Ranjan M, Boutet A, et al. Imaging alone versus microelectrode recording-guided targeting of the STN in patients with Parkinson's disease. J Neurosurg. 2018. https://doi.org/10.3171/ 2018.2.JNS172186.

41. Chen CC, Pogosyan A, Zrinzo LU, et al. Intra-operative recordings of local field potentials can help localize the subthalamic nucleus in Parkinson's disease surgery. Exp Neurol. 2006;198:214-21. 
42. Lambert C, Zrinzo L, Nagy Z, et al. Confirmation of functional zones within the human subthalamic nucleus: patterns of connectivity and sub-parcellation using diffusion weighted imaging. Neuroimage. 2012;60:83-94.

43. Plantinga BR, Temel Y, Duchin Y, et al. Individualized parcellation of the subthalamic nucleus in patients with Parkinson's disease with 7T MRI. Neuroimage. 2018;168:403-11.

44. Geraedts VJ, van Ham RAP, Marinus J, et al. Intraoperative test stimulation of the subthalamic nucleus aids postoperative programming of chronic stimulation settings in Parkinson's disease. Parkinsonism Relat Disord. 2019;65:62-6.

45. Nakajima T, Zrinzo L, Foltynie T, et al. MRI-guided subthalamic nucleus deep brain stimulation without microelectrode recording: can we dispense with surgery under local anaesthesia? Stereotact Funct Neurosurg. 2011;89:318-25.

46. Fluchere F, Witjas T, Eusebio A, et al. Controlled general anaesthesia for subthalamic nucleus stimulation in Parkinson's disease. J Neurol Neurosurg Psychiatry. 2014;85:1167-73.

47. Tsai ST, Chen TY, Lin SH, Chen SY. Five-year clinical outcome of local versus general anesthesia deep brain stimulation for Parkinson's disease. Parkinsons Dis. 2019. https://doi.org/10.1155/2019/ 5676345 .
48. Aygun D, Dere UA, Yildiz O, Temel Y, Kocabicak E. Characterizing the intraoperative microelectrode recording-induced microlesion effect on motor symptoms in patients with Parkinson's disease undergoing deep brain stimulation of the subthalamic nucleus. Turk Neurosurg. 2019;29:430-3.

49. Holewijn RA, Verbaan D, de Bie RMA, SChuurman PR. General anesthesia versus local anesthesia in stereotaxy (GALAXY) for Parkinson's disease: study protocol for a randomized controlled trial. Trials. 2017;18:417. https://doi.org/10.1186/s13063-0172136-8.

50. Kocabicak E, Aygun D, Alptekin O, et al. Conversion of local anesthesia-guided deep brain stimulation of the subthalamic nucleus to general anesthesia. J Neurol Surg Cent Eur Neurosurg. 2013;74:332-4.

51. Contarino MF, Bot M, Speelman JD, et al. Postoperative displacement of deep brain stimulation electrodes related to lead-anchoring technique. Neurosurgery. 2013;73:681-8.

52. Morishita T, Hilliard JD, Okun MS, et al. Postoperative lead migration in deep brain stimulation surgery: incidence, risk factors, and clinical impact. PLoS One. 2017;12:e0183711. https://doi.org/10. 1371/journal.pone.0183711. 